\title{
Evaluation of the Malignant Grade of Thymic Epithelial Tumors According to the Epithelial Subclassification
}

\author{
Satoshi Yoneda $^{1}$, Katsunobu Kawahara ${ }^{1}$, Kan Okabayashi $^{1}$, Takeshi Shiraishi ${ }^{1}$, Akinori Iwasaki ${ }^{1}$, \\ Takayuki Shirakusa ${ }^{1}$, Junji Kohno ${ }^{2}$, and Masahiro Kikuchi ${ }^{3}$ \\ ${ }^{1}$ Second Department of Surgery and ${ }^{3}$ First Department of Pathology, School of Medicine, Fukuoka University, 7-45-1 Nanakuma, Jonan-ku, \\ Fukuoka 814-80, Japan \\ ${ }^{2}$ Department of Pulmonary Surgery, National Sanatrium, Fukuoka Higashi Hospital, Fukuoka, Japan
}

\begin{abstract}
We investigated the clinicopathological correlations among 49 surgically resected thymic epithelial tumors (TET), which were subclassified according to the six subtypes established by the Marino, Kirchner, and Müller-Hermelink system, which were renamed as follows: spindle cell type (medullary thymoma), mixed spindle and polygonal cell type (mixed medullary and cortical thymoma), small polygonal cell type (predominantly cortical thymoma), large polygonal cell type (cortical thymoma), atypical type (well differentiated thymic carcinoma), and cytologically malignant type (highgrade thymic carcinoma). The related categories were grouped for statistical analysis as follows: group 1, spindle cell type and mixed type; group 2, small polygonal cell type and large polygonal cell type; group 3, atypical type; group 4, cytologically malignant type. The association of each group with the presence of myasthenia gravis, tumor stage, and the length of survival was studied. Myasthenia gravis was significantly present in patients with small polygonal type, large polygonal type, and atypical type tumors (groups 2 and 3) ( $P$ $=0.003)$. The tumors in group 1 showed the lowest tumor stage while those of group 4 had the most advanced tumor stage $(P=0.002)$. The patients in group 4 had the worst prognosis, followed by those in group 3,2 , and 1 , in that order. The differences among these groups were statistically significant $(P=0.0003)$. From our results, we determined that TET can be separated into an extremely low-grade malignancy group (group 1), a low-grade malignancy group (group 2), an intermediate malignancy group (group 3), and a high-grade malignancy group (group 4).
\end{abstract}

Key Words: thymic epithelial tumor, thymoma, thymic carcinoma, malignant grade, clinicopathologic classification

Reprint requests to: S. Yoneda

(Received for publication on June 15, 1998; accepted on May 27, 1999)

\section{Introduction}

Several histological classifications of thymic epithelial tumors have so far been proposed, although no definitive one has yet been published in the World Health Organization series of Histological Typing of Tumors. ${ }^{1}$ The establishment of an adequate classification system remains difficult, due to the functional, histogenetic, and morphologic complexity of these tumors.

Traditionally, thymomas have been histologically classified into four categories - predominantly spindle cell, predominantly lymphocytic, predominantly mixed lymphocytic and epithelial, and predominantly epithelial thymoma - based on the lymphocyte to epithelial cell ratio established by Lattes $^{2}$ and Bernatz et al. ${ }^{3}$ On the other hand, Marino, Kirchner, and MüllerHermelink $^{4,5}$ proposed a new morphologic classification of thymic epithelial tumors based on the resemblance of the neoplastic cells to the subtypes of normal thymic epithelial cells. They thus defined six categories: medullary thymoma, mixed medullary and cortical thymoma, predominantly cortical (organoid) thymoma, cortical thymoma, well-differentiated thymic carcinoma, and high-grade carcinoma (M-K-M system).

We classified 49 thymic epithelial tumors according to the M-K-M system but the categories were renamed as spindle cell type, mixed spindle cell and polygonal cell type, small polygonal cell type, large polygonal cell type, atypical type, and cytologically malignant type, respectively. In addition, we also studied such clinical data as stage classification, associated diseases, and survival, on 49 patients, to clarify the relationship between this subclassification and the clinical features.

\section{Materials and Methods}

We examined forty-nine thymic epithelial tumors which had been surgically resected in the Second Department 
of Surgery, Fukuoka University, and the Department of Pulmonary Surgery, Fukuoka Higashi Hospital, from 1979 to 1997. All the H\&E-stained slides were reviewed, and the thymic epithelial tumors were classified according to the M-K-M system but under the category names described above. The spindle cell type corresponded to medullary thymoma, which is composed of spindle-shaped epithelial cells with elongated nuclei, dispersed chromatin, and inconspicuous nucleoli (Fig. 1A). The mixed spindle cell and polygonal cell type corresponded to mixed corticomedullary thymoma in the M-K-M system (Fig. 1B). The small polygonal cell type corresponded to predominantly cortical (organoid) thymoma, which has small pale nuclei and is admixed with abundant lymphocytes (Fig. 1C). The large polygonal cell type corresponded to cortical thymoma, which is composed of cells with large pale nuclei with distinct nucleoli admixed with a moderate number of lymphocytes (Fig. 1D). The atypical type corresponded to well-differentiated thymic carcinoma, which grows in a solid and lobular pattern and is composed of polygonal cells with coarse chromatin, inconspicuous nucleoli, atypia and mitoses, and few or no lymphocytes (Fig. $1 \mathrm{E})$. The cytological malignant type corresponded to high-grade thymic carcinoma in the M-K-M system (Fig. 1F).

The clinicopathological stage was assessed according to the criteria proposed by Masaoka et al. ${ }^{6}$ The small number of cases for each histological type made the statistical analysis difficult, and thus the tumors were grouped for analysis as shown in Table 2: group 1, spindle cell type and mixed type; group 2 , small polygonal cell type and large polygonal cell type; group 3, atypical type; group 4, cytologically malignant type.

The correlation between the tumor type and the clinical data was analyzed by Fisher's exact test or the chisquared test. The influence of any prognostic factors on survival was analyzed using the Kaplan-Meier method, and comparisons of the survival curves were made using the log-rank test.

\section{Results}

The clinical data and histological subclassification are summarized in Table 1 . The patients comprised 22 males $(45 \%)$ and 27 females (55\%) ranging in age from 26 to 81 years (mean age 53.3 years). There were 5 spindle cell-type cases $(10 \%), 5$ mixed-type cases (10\%), 7 small polygonal cell-type cases (14\%), 9 large polygonal cell-type cases (19\%), 7 atypical-type cases $(14 \%)$, and 16 cytological malignant-type cases (33\%). Eight patients (16\%) presented with symptoms related to myasthenia gravis. Myasthenia gravis was present in groups 2 and 3 but not in groups 1 and $4(P=0.003)$ (Table 2). No other paraneoplastic diseases were present in any type.

Table 1. Clinicopathological features of the 49 patients with thymic epithelial tumors

\begin{tabular}{lr}
\hline & No. of cases $(\%)$ \\
\hline Age (years) & $26-81$ (mean 53.3) \\
Gender & $22(45)$ \\
Male & $27(55)$ \\
Female & \\
Stage & $14(29)$ \\
I & $8(16)$ \\
II & $20(41)$ \\
III & $7(14)$ \\
IV & \\
Myasthenia gravis & $8(16)$ \\
(+) & $41(84)$ \\
(-) & \\
Histology & $5(10)$ \\
Spindle cell type & $5(10)$ \\
Mixed type & $7(14)$ \\
Small polygonal cell type & $9(18)$ \\
Large polygonal cell type & $7(14)$ \\
Atypical type & $16(33)$ \\
Cytologically malignant type & $9(18)$ \\
Squamous cell carcinoma & $1(2)$ \\
Adenocarcinoma & $2(4)$ \\
Undifferentiated carcinoma & $1(2)$ \\
Small cell carcinoma & \\
\hline
\end{tabular}

Fig. 1. A A thymic epithelial tumor of spindle cell type. The tumor is composed of spindle-shaped epithelial cells. The type of tumor corresponds to predominantly spindle thymoma in the traditional system or medullary thymoma in the M-K-M system. B A thymic epithelial tumor of mixed type. The tumor is composed of spindle epithelial cells and polygonal cells. C A thymic epithelial tumor of small polygonal cell type. The tumor corresponds to predominantly lymphocytic thymoma based on the traditional system or predominantly cortical (organoid) thymoma according to the M-K-M system. The tumor cells have small pale nuclei and are admixed with abundant lymphocytes. D A thymic epithelial tumor of large polygonal cell type. The tumor corresponds to predominantly mixed thymoma based on the traditional system or cortical thymoma according to the M-K-M system. The tumor is composed of polygonal cells with large pale nuclei with distinct nucleoli, and are admixed with a moderate number of lymphocytes. $\mathbf{E}$ A thymic epithelial tumor of atypical type. The tumor is composed of polygonal cells with distinct cytoplasmic borders, with minimal nuclear atypia, and very few lymphocytes. This tumor corresponds to predominantly epithelial thymoma based on the traditional system or well-differentiated thymic carcinoma according to the M-K-M system. F A thymic epithelial tumor of cytological malignant type. The tumor corresponds to high-grade carcinoma according to the M-K-M system 

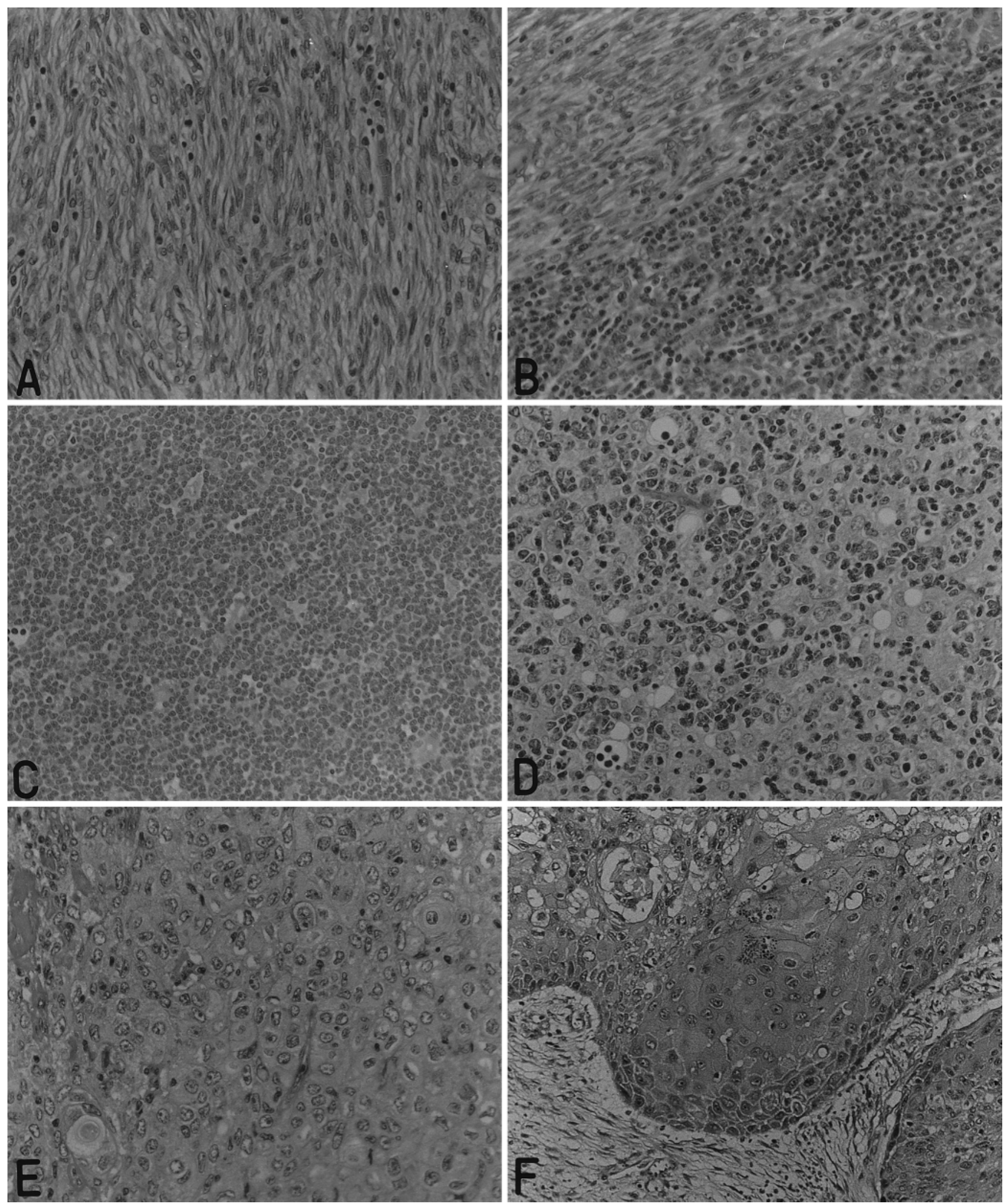
Table 2. Correlation between myasthenia gravis and the histological groups

\begin{tabular}{lccc}
\hline & MG $(+)$ & MG $(-)$ & Total \\
\hline $\begin{array}{l}\text { Group 1. Spindle cell type and } \\
\text { mixed type }\end{array}$ & 0 & 10 & 10 \\
$\begin{array}{l}\text { Group 2. Small polygonal cell type } \\
\quad \text { and large polygonal cell type }\end{array}$ & 7 & 9 & 16 \\
Group 3. Atypical type & 1 & 6 & 7 \\
Group 4. Cytologically malignant type & 0 & 16 & 16 \\
Total & 8 & 41 & 49 \\
\hline
\end{tabular}

MG, myasthenia gravis

$P=0.003$

Table 3. Correlation between tumor stage and the histological groups

\begin{tabular}{lrrrrrr}
\hline & \multicolumn{3}{c}{ Tumor stage } & \\
\cline { 2 - 5 } & I & II & III & IV & Total \\
\hline Group 1. Spindle cell type and mixed type & 8 & 2 & 0 & 0 & 10 \\
Group 2. Small polygonal cell type and & 6 & 3 & 6 & 1 & 16 \\
$\quad$ large polygonal cell type & & & & & 7 \\
Group 3. Atypical type & 0 & 1 & 10 & 5 & 16 \\
Group 4. Cytologically malignant type & 14 & 8 & 20 & 7 & 49 \\
Total & & & & & \\
\hline
\end{tabular}

$P=0.002$

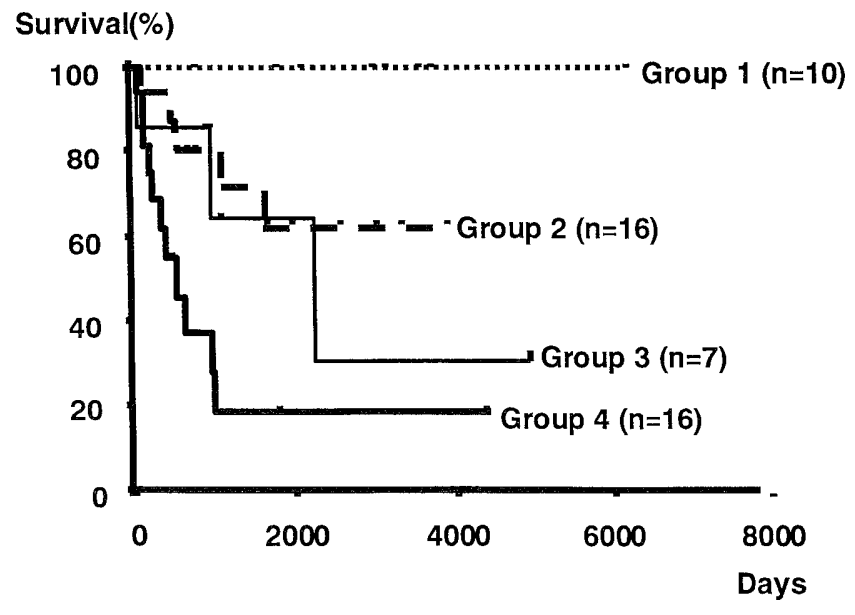

Fig. 2. Postoperative survival of 49 patients with thymic epithelial tumors. Group 1 comprises spindle cell and mixed type; group 2, small and large polygonal cell type; group 3; atypical type; group 4 , cytologically malignant malignant type. $P=$ 0.0003 by log rank test

The tumors were staged according to the criteria of Masaoka et al. ${ }^{6}$ Fifteen were stage I, 8 stage II, 20 stage III, and 7 stage IV. The correlation between tumor stage and histological group is shown in Table 3. Highly significant differences $(P=0.002)$ were observed regarding the stage distribution among groups: the tumors in group 1 had the lowest tumor stage, whereas those in group 4 showed the most advanced tumor stage.

The calculated postoperative survival curves are shown in Fig. 2. The group 4 patients had the worst prognosis (mean survival time $=1163.7 \pm 445.5$ days). The results were improved upon by group 3 (2484.3 \pm 858.7 days), group 2 (2745.9 \pm 418.2 days), and group 1 (6189 days) in that order. The differences among them were statistically significant $(P=0.0003)$.

\section{Discussion}

Thymic epithelial tumors are neoplasms arising from thymic epithelial cells with a variable ratio of associated nonneoplastic lymphoid cells. Therefore, a classification system of these tumors should be established based on the character of the epithelial tumor cells, but not based on the ratio of intermingled lymphocytes. ${ }^{7}$ The M-K-M system could be regarded as an adequate and reasonable classification system based on this definition of the tumors. However, the term "well-differentiated thymic carcinoma," which was proposed by Kirchner et al. to denote organotypical low-grade carcinoma, remains controversial. Quintanilla-Martines et al. ${ }^{8}$ defended this term by describing the characteristics of well-differentiated thymic carcinoma, i.e., cytologic atypia, invasiveness, high risk of recurrence, and the 
potential for metastases. These characteristics, however, might have been present in other types of thymoma, particularly cortical thymoma. Moreover, Ito et al. ${ }^{9}$ and Chan et al. ${ }^{10}$ reported that the infiltrating lymphocytes accompanying medullary and mixed thymoma are more mature, whereas those that accompany organoid thymoma, cortical thymoma, and well-differentiated thymic carcinoma are predominantly immature thymocytes. Shimosato ${ }^{1}$ also reported that thymic carcinoma does not contain any immature thymocytes, and is not associated with myasthenia gravis. These authors therefore suggested the descriptive terms "atypical" or "aggressive" to describe this thymoma because the malignancy of well-differentiated thymic carcinoma is intermediate between usual thymomas and thymic carcinomas. Recently, however, a small number of cases of thymic carcinoma arising in thymoma has been reported. ${ }^{11-13}$ Suster and Moran ${ }^{14}$ reported 22 cases of primary thymic epithelial neoplasms in which areas bearing the classic histological features of thymoma were found in conditions admixed with areas showing features of thymic carcinoma within the same tumor mass. They considered that all thymomas should be regarded as potentially malignant neoplasms, with the mature, well-circumscribed, and encapsulated thymoma at one end of the spectrum being equivalent to an in situ malignancy of the thymus, atypical thymoma representing an intermediate step in biologic and phenotypic differentiation, and thymic carcinoma representing the opposite end of the spectrum. Therefore, thymic epithelial tumors could be divided into an extremely lowgrade malignancy group (group 1, spindle cell type and mixed type), a low-grade malignancy group (group 2, small polygonal cell type and large polygonal cell type), an intermediate malignancy group (group 3, atypical type), and a high-grade malignancy group (group 4, cytologically malignant type) based on both our and previous gradings of the clinicopathological malignancy of these tumors. A comparison between the traditional and the M-K-M histological classification system is listed in Table 4. It is interesting to note that MüllerHermelink and colleagues ${ }^{4,5}$ and Quintanilla-Martines et $a .^{8}$ have reported that both medullary and mixed thymoma behave as benign tumors. In our study, all patients with these types of tumors showed a similarly excellent prognosis. However, one of five medullary (spindle cell type) thymomas in our series was microinvasive. Furthermore, there have also been reports of mediastinal recurrence or metastasis of this type of tumor. ${ }^{1,7,15}$ Medullary thymomas are generally innocuous, but are not absolutely benign. ${ }^{15}$ Thus the term "extremely low-grade malignancy," used here, more adequately describes these tumors than the term "benign." The extremely low-grade malignancy group corresponds to predominantly spindle cell thymoma and predominantly mixed (polygonal and spindle cell) thymoma in the traditional system, and to medullary and mixed corticomedullary thymoma in the M-K-M system. The low-grade malignancy group corresponds to predominantly mixed (lymphocytic and epithelial) thymoma, predominantly lymphocytic thymoma, and predominantly epithelial thymoma without atypia in the traditional system, and to organoid thymoma and cortical thymoma in the M-K-M system. The intermediate malignancy group (atypical type) corresponds to predominantly epithelial thymoma with atypia in the traditional system, and to well-differentiated thymic carcinoma in the M-K-M system.

Recently, Dorfman et al. ${ }^{16}$ reported a high expression of CD5 to be observed in the epithelial cells of overt thymic carcinoma, particularly those of squamous cell carcinoma, but not in those of well-differentiated thymic carcinoma and that, taken together, CD5 and CD99 (MIC2; O13, or other immature T-cell markers such as

Table 4. Malignant grade according to the prognosis of patients with thymic epithelial tumors, and the correlation between the malignant grade and both the traditional and M-K-M classification system

Traditional system Marino, Kirchner, and Müller-Hermelink system

Extremely low-grade malignancy group (spindle cell type and mixed spindle and polygonal cell type) Predominantly spindle cell thymoma Predominantly mixed (lymphocytic and spindle) thymoma Medullary thymoma

Mixed medullary and cortical thymoma

Low-grade malignancy group (small polygonal cell type and large polygonal cell type) Predominantly lymphocytic thymoma

Predominantly mixed (lymphocytic and polygonal) thymoma

Predominantly epithelial thymoma (without atypia)

Predominantly cortical (organoid) thymoma Cortical thymoma

Intermediate malignancy group (atypical type) Predominantly epithelial thymoma (with atypia)

Well-differentiated thymic carcinoma

High-grade malignancy group (cytologically malignant type) Thymic carcinoma

High-grade thymic carcinoma 
TdT and CD1a) may be useful in the differential diagnosis between well-differentiated thymic carcinoma and true thymic carcinoma.

A standardized approach to the classification of thymic epithelial tumors is therefore indispensable in order to both avoid a misdiagnosis and also elucidate the essence of these unique tumors.

Acknowledgments. The authors are grateful to Prof. Hans-Konrad Müller-Hermelink and Prof. Alexander Marx (Institute of Pathology, University of Würzburg, Würzburg, Germany) for assistance with the thymic epithelial tumor classification system, and to Mr. Kensaku Satou for his valuable help in performing the statistical analysis in this study.

\section{References}

1. Shimosato Y (1994) Controversies surrounding the subclassification of thymoma. Cancer 74:542-544

2. Lattes R (1962) Thymoma and other tumors of the thymus. An analysis of 107 cases. Cancer 15:1224-1260

3. Bernatz PE, Harrison EG, Glagett OT (1961) Thymoma. A clinico-pathologic study. J Thorac Cardiovasc Surg 42:424-444

4. Marino M, Müller-Hermelink HK (1985) Thymoma and thymic carcinoma. Relation of thymic epithelial cells to the cortical and medullary differentiation of thymus. Virchows Arch A 407:119149

5. Kirchner T, Schalke B, Marx A, Müller-Hermelink HK (1989) Evaluation of prognostic features in thymic epithelial tumors. Thymus 14:195-203
6. Masaoka A, Monden Y, Nakahara K, Tanioka T (1981) Followup study of thymoma with special reference to their clinical stages. Cancer 48:2485-2492

7. Levine GD, Rosai J (1978) Thymic hyperplasia and neoplasia. A review of current concepts. Hum Pathol 9:495-515

8. Quintanilla-Martines L, Wilkins EW Jr, Choi N, Efird J, Hug E, Harris NL (1994) Thymoma: histologic subclassification is an independent prognostic factor. Cancer 74:606-617

9. Ito M, Taki T, Miyake M, Mitsuoka A (1988) Lymphocyte subsets in human thymoma studied with monoclonal antibodies. Cancer 61:284-287

10. Chan JKC, Tsang WYW, Senevieatne S, Pau MY (1995) The MIC2 antibody O13: practical application for the study of thymic epithelial tumors. Am J Surg Pathol 19:1115-1123

11. Shimosato Y, Kameya T, Nagaki K, Suemasu K (1977) Squamous cell carcinoma of the thymus: an analysis of eight cases. Am J Surg Pathol 1:109-121

12. Wick MR, Weiland LH, Scheithauer BZ, Bernatz PE (1982) Primary thymic carcinomas. Am J Surg Pathol 6:613630

13. Kuo TT, Chang JP, Lin FJ, Wu WC, Chang CH (1990) Thymic carcinomas: histological varieties and immunohistochemical study. Am J Surg Pathol 14:24-34

14. Suster S, Moran CA (1996) Primary thymic epithelial neoplasms showing combined features of thymoma and thymic carcinoma. A clinicopathologic study of 22 cases. Am J Surg Pathol 20:14691480

15. Pan CC, Wu HP, Yang CF, Chen YK, Chiang H (1994) The clinicopathological correlation of epithelial subtyping in thymoma: a study of 112 consecutive cases. Hum Pathol 25:893899

16. Dorfman DM, Shahsafaei A, Chan JKC (1997) Thymic carcinomas, but not thymomas and carcinomas of other sites, show CD5 immunoreactivity. Am J Surg Pathol 21:936-940 